\title{
A NEW METHOD OF INVERSION OF THE LAPLACE TRANSFORM*
}

\author{
BY \\ ATHANASIOS PAPOULIS \\ Polytechnic Institute of Brooklyn
}

Introduction. In determining a function $r(t)$ from its Laplace transform $R(p)$

$$
R(p)=\int_{0}^{\infty} e^{-p t} r(t) d t
$$

one applies either a partial fraction expansion or an integration along some contour in the complex $p$-plane; one thus obtains $r(t)$ in terms of the poles and residues of $R(p)$, or from the values of $R(p)$ on a contour of the $p$-plane. Both methods have obvious disadvantages for a numerical analysis.

In the following we propose to develop a method for determining $r(t)$ in terms of the values of $R(p)$ on an infinite sequence of equidistant points

$$
p_{k}=a+k \sigma \quad k=0,1, \cdots, n, \cdots
$$

on the real $p$-axis, where $a$ is a real number in the region of existence of $R(p)$, and an arbitrary positive integer. That $R(p)$ is uniquely determined from its values at the above points, is known [1]. It should therefore be possible to express $r(t)$ directly in terms of $R(a+k \sigma)$. In this paper it will be shown that $r(t)$ can be written in the form

$$
r(t)=\sum_{k=0}^{\infty} C_{k} \varphi_{k}(t),
$$

where the $\varphi_{k}$ 's are known functions, and the constants $C_{k}$ can readily be determined from the values of $R(p)$ at the points $a+k \sigma$.

The $\varphi_{k}$ 's can be chosen from several sets of complete orthogonal functions; in our discussion we shall use the familiar trigonometric set, the Legendre set and the Laguerre polynomials.

The trigonometric set. We introduce the variable $\theta$ defined by

$$
e^{-\sigma t}=\cos \theta \quad \sigma>0 .
$$

The $(0, \infty)$ interval transforms into the interval $(0, \pi / 2)$, and $r(t)$ becomes

$$
r\left(-\frac{1}{\sigma} \ln \cos \theta\right) \text {. }
$$

For simplicity of notation we shall denote the above function by $r(\theta)$ using the same letter $r$.

The defining equation (1) takes the form

$$
\sigma R(p)=\int_{0}^{\pi / 2}(\cos \theta)^{(p / \theta)-1} \sin \theta r(\theta) d \theta
$$

*Received January 6, 1956. Part of a paper presented at the Symposium on Modern Network Synthesis, Polytechnic Institute of Brooklyn, April 1955. 
hence with

we have

$$
p=(2 k+1) \sigma \quad k=0,1,2, \cdots
$$

$$
\sigma R[(2 k+1) \sigma]=\int_{0}^{\pi / 2}(\cos \theta)^{2 k} \sin \theta r(\theta) d \theta .
$$

In the following we shall assume, without loss of generality, that $r(0)=0$ subtracting, if necessary, a constant from $r(\theta)$. The function $r(\theta)$ can be expanded in the $(0, \pi / 2)$ interval into an odd-sine series

$$
r(\theta)=\sum_{k=0}^{\infty} C_{k} \sin (2 k+1) \theta
$$

This can of course be done by properly extending the definition of $r(\theta)$ in the $(-\pi,+\pi)$ interval.

We shall next determine the coefficients $C_{k}$. We have

$$
(\cos \theta)^{2 n} \sin \theta=\left(\frac{e^{i \theta}+e^{-i \theta}}{2}\right)^{2 n} \frac{e^{i \theta}-e^{-j \theta}}{2 j},
$$

expanding in the right hand side and properly collecting terms we obtain $2^{2 n}(\cos \theta)^{2 n} \sin \theta=\sin (2 n+1) \theta+\cdots$

$$
+\left[\left(\begin{array}{c}
2 n \\
k
\end{array}\right)-\left(\begin{array}{c}
2 n \\
k-1
\end{array}\right)\right] \sin [2(n-k)+1] \theta+\cdots+\left[\left(\begin{array}{c}
2 n \\
n
\end{array}\right)-\left(\begin{array}{c}
2 n \\
n-1
\end{array}\right)\right] \sin \theta .
$$

We next insert (7) and (8) into (6); because of the orthogonality of the odd sines in the $(0, \pi / 2)$ interval and since

$$
\int_{0}^{\pi / 2}[\sin (2 n+1) \theta]^{2} d \theta=\frac{\pi}{4}
$$

we have

$$
\begin{aligned}
\sigma R[(2 n+1) \sigma]=2^{-2 n} \frac{\pi}{4}\left\{\left[\left(\begin{array}{c}
2 n \\
n
\end{array}\right)-\left(\begin{array}{c}
2 n \\
n-1
\end{array}\right)\right]\right. & C_{0}+\cdots \\
& \left.+\left[\left(\begin{array}{c}
2 n \\
k
\end{array}\right)-\left(\begin{array}{c}
2 n \\
k-1
\end{array}\right)\right] C_{n-k}+\cdots+C_{n}\right\}
\end{aligned}
$$

hence with $n=0,1,2, \cdots$ we obtain the system

$$
\begin{gathered}
\frac{4}{\pi} \sigma R(\sigma)=C_{0} \\
2^{2} \frac{4}{\pi} \sigma R(3 \sigma)=C_{0}+C_{1} \\
\ldots \ldots \\
2^{2 n} \frac{4}{\pi} \sigma R[(2 n+1) \sigma]=\left[\left(\begin{array}{c}
2 n \\
n
\end{array}\right)-\left(\begin{array}{c}
2 n \\
n-1
\end{array}\right)\right] C_{0} \\
+\cdots \\
+\left[\left(\begin{array}{c}
2 n \\
k
\end{array}\right)-\left(\begin{array}{c}
2 n \\
k-1
\end{array}\right)\right] C_{n-k}+\cdots+C_{n} .
\end{gathered}
$$


Thus $R(\sigma)$ gives $C_{0}, R(3 \sigma)$ give $C_{1}$ and each value of $R(p)$ at the points $(2 k+1) \sigma$ together with the coefficients $C_{0}, C_{1} \ldots, C_{k-1}$, determines $C_{k}$. The system (9) can obviously be written in such a way as to give directly $C_{k}$ in terms of $R(\sigma), R(3 \sigma), \ldots$ alone, but not much is gained, since in a numerical evaluation of the $C_{k}$ 's equation (9) can be used as easily. Table 1 gives the numerical values of the coefficients of the $C_{k}$ 's in the right hand side of $(9)$, for $k=0,1, \cdots, 10$.

TABLE 1

\begin{tabular}{|c|c|c|c|c|c|c|c|c|c|c|c|}
\hline$n$ & $C_{0}$ & $C_{1}$ & $C_{2}$ & $C_{8}$ & $C_{4}$ & $C_{5}$ & $C_{6}$ & $C_{7}$ & $C_{8}$ & $C_{0}$ & $C_{10}$ \\
\hline 0 & 1 & & & & & & & & & & \\
\hline 1 & 1 & 1 & & & & & & & & & \\
\hline 2 & 2 & 3 & 1 & & & & & & & & \\
\hline 3 & 5 & 9 & 5 & 1 & & & & & & & \\
\hline 4 & 19 & 28 & 20 & 7 & 1 & & & & & & \\
\hline 5 & 42 & 90 & 75 & 35 & 9 & 1 & & & & & \\
\hline 6 & 132 & 297 & 275 & 154 & 54 & 11 & 1 & & & & \\
\hline 7 & 429 & 1001 & 1001 & 637 & 273 & 77 & 13 & 1 & & & \\
\hline 8 & 1430 & 3432 & 3640 & 2548 & 1260 & 440 & 104 & 15 & 1 & & \\
\hline 9 & 4862 & 11934 & 13260 & 9996 & 5508 & 2244 & 663 & 135 & 17 & 1 & \\
\hline 10 & 16796 & 41990 & 48450 & 38760 & 23256 & 10659 & 3705 & 950 & 170 & 19 & 1 \\
\hline
\end{tabular}

Thus a method of analysis has resulted which compares sometimes favorably with the known methods of numerical evaluation of $r(t)$. Indeed the computation of $R((2 k+1) \sigma)$ presents no difficulty, and the $C_{k}$ 's can be readily determined from (9); the trigonometric functions are available, hence $r(\theta)$ can be computed with any desired accuracy from the series (7). In a numerical evaluation of $r(\theta)$ one computes the finite sum

$$
r_{N}(\theta)=\sum_{k=0}^{N} C_{k} \sin (2 k+1) \theta
$$

of the first $N+1$ terms of (7); as $N$ tends to infinity $r_{N}(\theta)$ tends to $r(\theta)$. The nature of the approximation is well known from the theory of Fourier series [2]; $r_{N}(\theta)$ and $r(\theta)$ are related by the equation

$$
r_{N}(\theta)=\frac{4}{\pi} \int_{0}^{\pi / 2} r(y) \frac{\sin \left[\frac{1}{2}(4 N+3)(\theta-y)\right]}{\sin \frac{1}{2}(\theta-y)} d y,
$$

thus the approximating function $r_{N}(\theta)$ is the average of $r(\theta)$ with the Fourier kernel

$$
\frac{\sin \left[\frac{1}{2}(4 N+3)(\theta-y)\right]}{\sin \frac{1}{2}(\theta-y)}
$$

as the weighting factor. From $r(\theta)$ one can readily obtain $r(t)$ with the change of variable established by (4); however, Eq. (7) can be written directly in the time domain. Indeed since

$$
\frac{\sin n \theta}{\sin \theta}=U_{n}(x) \quad \cos \theta=x
$$


where $\ln (x)$ are the Tchebycheff sine-polynomials of order $n$ and

$$
\sin \theta=\left(1-e^{-2 \sigma t}\right)^{1 / 2}
$$

we have from (7)

$$
r(t)=\left(1-e^{-2 \sigma t}\right)^{1 / 2} \sum_{k=0}^{\infty} C_{k} U_{2 k}\left(e^{-\sigma t}\right) .
$$

The choice of $\sigma$ depends on the interval $(0, T)$ in which $r(t)$ is best to be described; if it is chosen so that

$$
e^{-\sigma T}=\frac{1}{2}
$$

then the $(0, T)$ interval transforms into the $(0, \pi / 3)$ interval. If a detailed description of $r(t)$ is desired both near the origin and for large values of $t$, then the function can be evaluated twice with two different values of $\sigma$.

The above provides a simple proof of the announced theorem that the Laplace transform $R(p)$ is uniquely determined from its values at the sequence

$$
p_{k}=a+k \sigma \quad k=0,1, \cdots, n, \cdots
$$

of equidistant points on the real $p$-axis. This proof uses the well-known orthogonality and completeness of the trigonometric set. Indeed $r(\theta)$, and hence $r(t)$, is completely determined from the coefficients $C_{k}$ of (7); these coefficients can be determined from $R(a+k \sigma)$; knowing $r(t)$ one clearly has $R(p)$ therefore $R(p)$ is uniquely determined from its values at the points (2).

The Legendre set. We shall next expand $r(t)$ into a series of Legendre polynomials. We introduce the logarithmic time-scale $x$ defined by

$$
e^{-\sigma t}=x \quad \sigma>0 .
$$

The $(0, \infty)$ interval transforms into the interval $(1,0)$ : again we shall denote the function

$$
r\left(-\frac{1}{\sigma} \ln x\right)
$$

by $r(x)$. Equation (1) takes the form

$$
\sigma R(p)=\int_{0}^{1} x^{(p / \sigma)-1} r(x) d x
$$

from which we obtain with $p=(2 k+1) \sigma$,

$$
\sigma R[(2 k+1) \sigma]=\int_{0}^{1} x^{2 k} r(x) d x .
$$

Thus the value of the function $R(p)$ at the point $[(2 k+1) \sigma]$ gives the $2 k$ th moment of the function $r(x)$ in the $(0,1)$ interval

It is known that the Legendre polynomials $P_{k}(x)$ form a complete orthogonal set in the $(-1,1)$ interval; We extend the definition of $r(x)$ in the $(-1,1)$ interval by making

$$
r(-x)=r(x) .
$$


This function, because of its evenness, can be expanded into a series of even Legendre polynomials. We thus have

$$
r(x)=\sum_{k=0}^{\infty} C_{k} P_{2 k}(x),
$$

using the time scale we can write (16) in the form

$$
r(t)=\sum_{k=0}^{\infty} C_{k} P_{2 k}\left(e^{-\sigma t}\right) .
$$

To determine the coefficients $C_{k}$ in (17) we observe that $P_{2 k}\left(e^{-\sigma t}\right)$, being an even polynomial in $e^{-\sigma t}$, of degree $2 k$, will have as transform the function

$$
\Phi_{2 k}(p)=\frac{N(p)}{p(p+2 \sigma) \cdots(p+2 k \sigma)},
$$

where $N(p)$ is a polynomial of degree less than $2 k$. It is further known that

$$
\int_{0}^{1} x^{2 n} P_{2 k}(x) d x=0 \text { for } n<k .
$$

From Eqs. (18) and (15) follows that

$$
\Phi_{2 k}[(2 n+1) \sigma]=0 \quad n=0,1, \cdots, k-1
$$

hence the roots of $N(p)$ are

$$
(2 n+1) \sigma \quad n=0,1, \cdots, k-1
$$

and $\Phi_{2 k}(p)$ can be written in the form

$$
\Phi_{2 k}(p)=\frac{(p-\sigma)(p-3 \sigma) \cdots[P-(2 k-1) \sigma]}{p(p+2 \sigma) \cdots(p+2 k \sigma)} A,
$$

where $A$ is a constant; to determine $A$ we observe from the initial value theorem that

$$
\lim _{p \rightarrow \infty} p \Phi_{2 k}(p)=A=P_{2 k}(1)
$$

and since $P_{2 k}(1)=1$, we must have

$$
A=1 .
$$

Thus the Laplace transform of $P_{2 k}\left(e^{-\sigma t}\right)$ is given by

$$
\Phi_{2 k}(p)=\frac{(p-\sigma)(p-3 \sigma) \cdots[p-(2 k-1) \sigma]}{p(p+2 \sigma) \cdots(p+2 k \sigma)} .
$$

Taking the transform of both sides of (17) we obtain

$$
R(p)=\frac{C_{0}}{p}+\sum_{k=1}^{\infty} \frac{(p-\sigma) \cdots[p-(2 k-1) \sigma]}{p \cdots(p+2 k \sigma)} C_{k} .
$$

If we replace $p$ by

$$
\sigma, 3 \sigma, \cdots,(2 k+1) \sigma, \cdots
$$


in Eq. (19), we obtain the system

$$
\begin{aligned}
\sigma R(\sigma) & =C_{0}, \\
\sigma R(3 \sigma) & =\frac{C_{0}}{3}+\frac{2 C_{1}}{3 \cdot 5}, \\
\text {. . . . . . . . . } & \text {. } \\
\sigma R[(2 k+1) \sigma] & =\frac{C_{0}}{2 k+1}+\frac{2 k C_{1}}{(2 k+1)(2 k+3)}+\cdots \\
& +\frac{2 k(2 k-2) \cdots 2 C_{k}}{(2 k+1)(2 k+3) \cdots(4 k+1)} .
\end{aligned}
$$

Again $R(\sigma)$ gives $C_{0}, R(3 \sigma) C_{1}$ and so on. The partial sum $r_{N}(x)$ is the average of $r(x)$ with the Legendre kernel as the weighting factor. The constant $\sigma$ is chosen with the same considerations as in $I$.

The above discussion furnishes a proof of the "Moment theorem" [1], [4]: that a function $r(x)$ in the $(0,1)$ interval is uniquely determined from its moments.

$$
M_{m}=\int_{0}^{1} r(x) x^{m} d x \quad m=0,1, \cdots .
$$

The proof is based on the orthogonality and completeness of the Legendre polynomials. In fact we also succeeded in writing $r(x)$ as an infinite sum of Legendre polynomials that can be determined from the moments of $r(x)$; these coefficients are given by the system (21) where on the left hand side we replace $R(2 k+1) \sigma)$ by $M_{2 k}$.

The Laguerre set. As a last case we shall consider the Laguerre set which has already been used in network analysis and synthesis [5]. The method described here will give a simpler way of determining the coefficients of the resulting expansion; it will also make clear the nature of the approximation, if the series contains only the first $N+1$ terms.

The usual definition of the Laguerre polynomials $L_{k}(t)$ is

$$
L_{k}(t)=e^{t} \frac{d^{k}}{d t^{k}}\left[\frac{t^{k}}{k !} e^{-t}\right]
$$

With

$$
\varphi_{k}(t)=e^{-t} L_{k}(t)
$$

we easily obtain for the transform of $\varphi_{k}(t)$

$$
\Phi_{k}(p)=\frac{p^{k}}{(p+1)^{k+1}}
$$

Since the derivatives of $\Phi_{k}(p)$ of order less than $k$ are zero at the origin, we must have [i]

$$
\int_{0}^{\infty} t^{n} \varphi_{k}(t) d t=0 \text { for } n \leqq k-1
$$

With

$$
r(t)=\sum_{k=0}^{\infty} C_{k} \varphi_{k}(t)
$$


we have

$$
R(p)=\sum_{k=0}^{\infty} C_{k} \frac{p^{k}}{(p+1)^{k+1}} .
$$

It can be shown by differentiating $n$ times the power series expansion at the origin of $1 /(p+1)$ that

$$
\frac{p^{k}}{(p+1)^{k+1}}=p^{k} \sum_{n=0}^{\infty}\left(\begin{array}{c}
n+k \\
k
\end{array}\right)(-1)^{n} p^{n} .
$$

Expanding the function $R(p)$ at the origin we obtain

$$
R(p)=\sum_{k=0}^{\infty} a_{k} p^{k} .
$$

From Eqs. (27), (28) and (29) we obtain equating equal powers of $p$

$$
\begin{aligned}
& a_{0}=C_{0} \\
& a_{1}=C_{1}-C_{0} \\
& \ldots \ldots \ldots \\
& a_{k}=C_{k}-\left(\begin{array}{l}
k \\
1
\end{array}\right) C_{k-1}+\cdots+(-1)^{k} C_{0} .
\end{aligned}
$$

The above system can be solved explicitly for $C_{k}$, with a simple induction [6]; the result is given by

$$
C_{k}=\sum_{i=0}^{k}\left(\begin{array}{c}
k \\
j
\end{array}\right) a_{k-i}
$$

Thus knowing the coefficients $a_{k}$ of the series expansion (29) of $R(p)$ we can readily determine from (31) the coefficients of (26).

Suppose that $r(t)$ is approximated by the finite sum

$$
r_{N}(t)=\sum_{k=0}^{N} C_{k} \varphi_{k}(t)
$$

of the first $N+1$ terms of (26); then the transforms $R_{N}(p)$ and $R(p)$ of $r_{N}(t)$ and $r(t)$ have equal derivatives at the origin of order up to $N$, therefore [7]

$$
\int_{0}^{\infty} t^{n} r_{N}(t) d t=\int_{0}^{\infty} t^{n} r(t) d t \quad n \leqq N
$$

that is the function $r(t)$ and $r_{N}(t)$ have equal moments of order up to $N$.

Examples. In the following applications we shall use for our expansions the trigonometric set. We have approximated the inverse of $R(p)$ by

$$
r_{N}(\theta)=\sum_{k=0}^{N} C_{k} \sin (2 k+1) \theta
$$

where the coefficients $C_{k}$ are given by (9) which we write in the form

$$
\frac{\pi}{4} C_{n}=\sigma 2^{2 n} R[(2 n+1) \sigma]-\sum_{j=0}^{n-1}\left[\left(\begin{array}{c}
2 n \\
j-1
\end{array}\right)-\left(\begin{array}{c}
2 n \\
n-j-1
\end{array}\right)\right] C_{i} .
$$


As examples we shall take functions whose inverse $r(t)$ is known, so as to compare $r(t)$ with $r_{N}(t)$. For the choice of $\sigma$ we are guided either by the $(0, T)$ interval of interest, or from the $(0, p)$ interval of the real $p$ axis in which $R(p)$ has its greatest variation; the choice of $\sigma$ is not critical.

Example 1.

$$
R(p)=\frac{\pi}{\pi} \frac{1}{(p+0.2)^{2}+1} \text { we take } \sigma=0.2
$$

TABLE 2

\begin{tabular}{rrr}
\hline & Example 1 & Example 2 \\
\cline { 2 - 4 } & \multicolumn{1}{c}{$C_{k} 10^{4}$} & $C_{k} 10^{4}$ \\
\hline 0 & 1724 & 1961 \\
1 & 3154 & 4899 \\
2 & 205 & 4009 \\
3 & -2075 & 460 \\
4 & 380 & 633 \\
5 & 530 & 1762 \\
6 & -754 & 166 \\
7 & 474 & 862 \\
8 & -193 & 718 \\
9 & -40 & 199 \\
10 & 58 & 982 \\
\hline
\end{tabular}

From equation (34) we obtain for the coefficients $C_{k}$ the numbers given in Table 2. These values inserted into (11) give for $r_{N}(\theta)$ at the points

$$
\theta=0,5, \cdots, 90^{\circ}
$$

the numbers in Table 3.

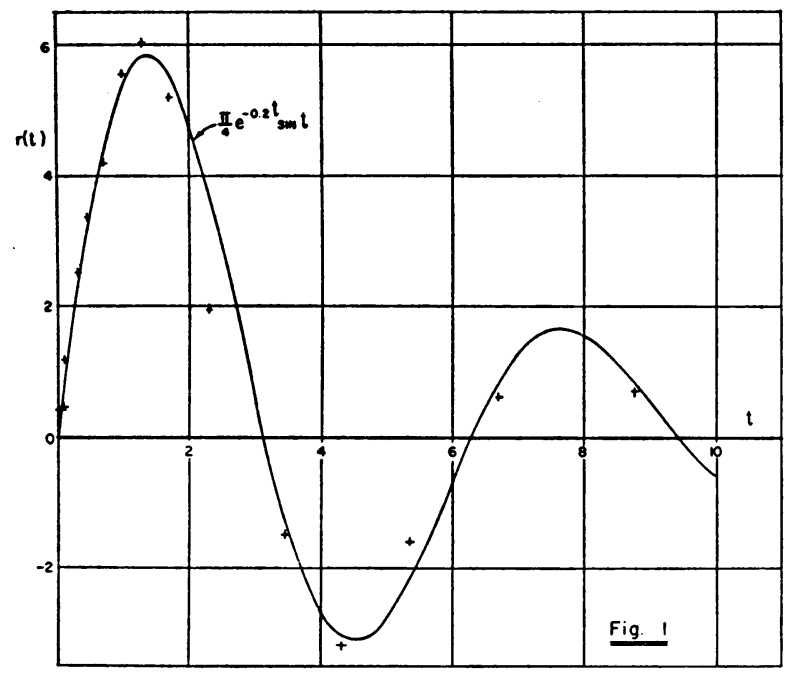


The curve of Fig. 1 gives the inverse

$$
r(t)=\frac{\pi}{4} e^{-0.2 t} \sin t
$$

of $R(p)$; the + points give the values of $r_{N}(t)$ as computed. The relationship between $\theta$ and $t$ is established in (4).

Example 2.

$$
R(p)=\frac{\pi}{4} \frac{1}{\left(p^{2}+1\right)^{1 / 2}} \quad \sigma=0.2
$$

This example is chosen because of its discontinuity at the origin; clearly since

$$
r(0) \neq 0
$$

$\mathbf{r}(\theta)$ will be discontinuous at $\theta=0$, and $r_{N}(\theta)$ will exhibit the Gibb's phenomenon.

TABLE 3

\begin{tabular}{rcc}
\hline & Example 1 & Example 2 \\
\hline \multicolumn{1}{c}{$\theta$} & $r_{N}(\theta) \times 10^{4}$ & $r_{N}(\theta) \times 10^{4}$ \\
\hline 5 & 398 & 8133 \\
10 & 432 & 8739 \\
15 & 1158 & 6958 \\
20 & 2511 & 7787 \\
25 & 3362 & 7896 \\
30 & 4215 & 6363 \\
35 & 5571 & 5977 \\
40 & 6029 & 5241 \\
45 & 5181 & 2612 \\
50 & 4048 & 615 \\
55 & 1944 & -834 \\
60 & -1502 & -3208 \\
65 & -3272 & -3190 \\
70 & -1590 & 286 \\
75 & 570 & 1748 \\
80 & 694 & -11 \\
85 & -33 & -412 \\
\hline
\end{tabular}

The values of $C_{k}$ and $r_{N}(\theta)$ are listed in Table 2 and Table 3. In Fig. 2 the inverse

$$
\frac{\pi}{4} J_{0}(t)
$$

is plotted; the + points give the computed values of $r_{N}(t)$.

We see from the above examples that $r_{N}(t)$ is a good approximation of $r(t)$.

The oscillation near $t=0$ of Example 2 could have been avoided and a better fitting obtained if instead of $R(p)$ the function

$$
R(p)-\frac{[p R(p)]_{p-\infty}}{p}=\frac{\pi}{4}\left(\frac{1}{\left(p^{2}+1\right)^{1 / 2}}-\frac{1}{p}\right)
$$


were chosen, since its inverse satisfies the condition

$$
r(0)=0 \text {. }
$$

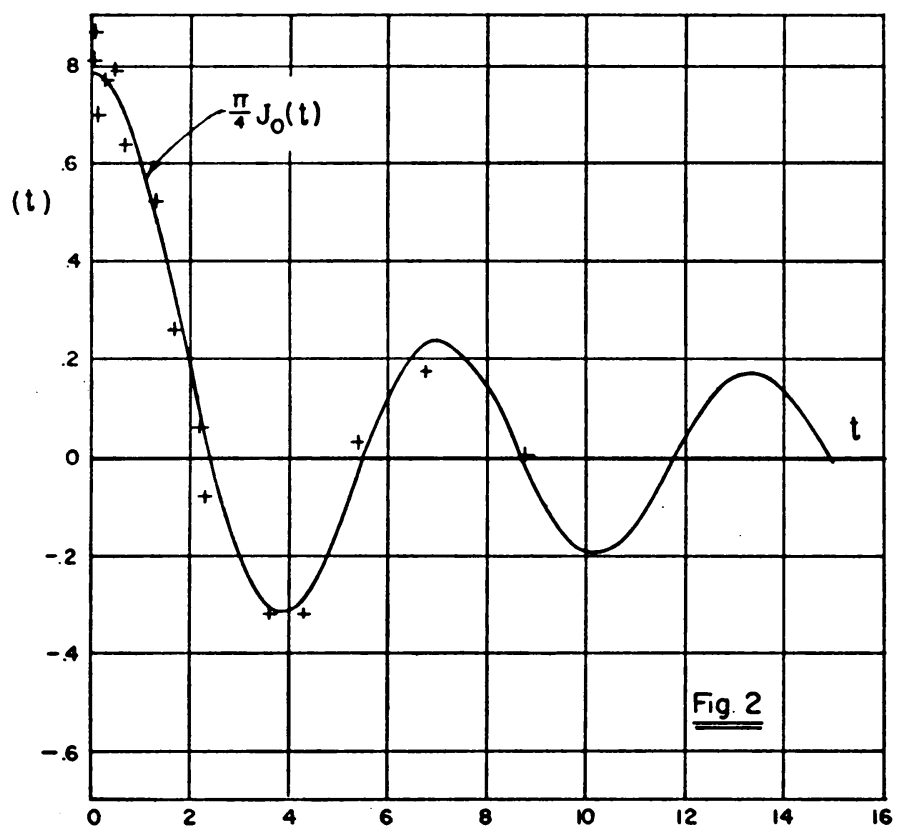

\section{REFERENCES}

1. G. Doetsch, Laplace transformation, Dover, 1943

2. E. A. Guillemin, The mathematics of circuit analysis, John Wiley \& Son, New York, 1947

3. R. Courant and D. Hilbert, Methoden der mathematischen Physik I, Springer, Berlin

4. D. V. Widder, The Laplace transform, Princeton University Press, 1946

5. W. H. Kautz, Transient synthesis in the time domain, Trans. IRE PGCT, Sept. 1954

6. G. Weiss, On the expansion of a function in terms of Laguerre polynomials, Trans. IRE, CT-2, p. 283, Sept. 1955

7. W. C. Elmore, The transient response of damped linear networks, J. Appl. Phys., Jan. 1948 